\title{
Accurate Detection of Helicobacter pylori Antigen in Human Stool Specimens by Two Novel Immunoassays
}

\author{
Ralf Ignatius $^{1,2 *}$, Christiane Berg ${ }^{1}$, Chris Weiland ${ }^{1}$, Angela Darmer ${ }^{1}$, Thilo Wenzel ${ }^{1}$, Marion Lorenz ${ }^{3}$, \\ Jörg Fuhrmann ${ }^{1}$ and Michael Müller ${ }^{1}$ \\ ${ }^{1}$ MVZ Labor 28, Mecklenburgische Str. 28, 14197 Berlin, Germany \\ ${ }^{2}$ Department of Microbiology and Infection Immunology, Charité - Universitätsmedizin Berlin, Campus Benjamin Franklin, \\ Hindenburgdamm 30, 12200 Berlin, Germany \\ ${ }^{3}$ R-Biopharm, An der neuen Bergstraße 17, 64297 Darmstadt, Germany
}

Received: 15 April 2019; accepted: 02 May 2019

\begin{abstract}
Stool antigen tests are recommended for the diagnosis of Helicobacter pylori infection. Here, we compared two novel assays, i.e., one enzyme immunoassay (EIA) and one immunochromatography assay (ICA), with a chemiluminescence immunoassay (CLIA) that had previously been compared with rapid urease test, histology, and urea breath test. Two hundred sixty-six frozen stool samples with defined CLIA results (42 positives, 219 negatives, and 5 samples with borderline results) collected between January and May 2018 were thawed and immediately tested by EIA, ICA, and CLIA.

In 248 samples with repeatedly positive/negative CLIA results, EIA and ICA were positive for 40 and 37 of 41 CLIA-positive samples and yielded negative results for 206 and 201 of 207 CLIA-negative samples, respectively. There was a high positive percent agreement (EIA, 97.6\%; 95\% confidence interval (95\% CI), 86.3-100\%; ICA, 90.2\%; 95\% CI, 76.9-96.7\%), as well as a negative percent agreement between the assays (EIA, 99.5\%; 95\% CI, 97.0-100\%; ICA, 97.1\%; 95\% CI, 93.7-98.8\%). This was further supported by kappa values indicating very good agreement (CLIA vs. EIA, 0.971; CLIA vs. ICA, 0.857). In conclusion, both EIA and ICA comprise valuable assays for the detection of $H$. pylori antigen in stool samples.
\end{abstract}

Keywords: Helicobacter pylori, stool antigen tests, enzyme immunoassay, immunochromatography assay, chemiluminescence immunoassay

\section{Introduction}

Since Helicobacter pylori infection may cause significant complications, e.g., peptic ulcer disease, carcinoma, or mucosa-associated lymphoid tissue lymphoma, its accurate diagnosis is relevant. Diagnostic tests consist of invasive tests, i.e., culture, histology, rapid urease test (RUT), and PCR, as well as non-invasive tests, such as urea breath test (UBT), serological assays for the detection of serum antibodies, and stool antigen tests (SATs) [1, 2].

SATs comprise enzyme immunoassays (EIAs) or immunochromatography assays (ICAs), detecting H. pylori antigens by the use of either monoclonal or polyclonal antibodies. In general, ICAs are easier to perform than EIAs, but previous studies have shown that EIAs are more accurate than ICAs [3, 4]. Furthermore, assays using monoclonal antibodies are more accurate than assays based on polyclonal antibodies [5]. In fact, SATs in the form of monoclonal EIAs have a pooled sensitivity of $94 \%$ and specificity of $97 \%$ [5], are less expensive than the UBT, and have therefore been recommended by international and national guidelines as alternatives to the UBT for both diagnosis of infection and confirmation of eradication following therapy [6-8]. SATs are particularly interesting for

*Author for correspondence: MVZ Labor 28, Mecklenburgische Str. 28, 14197 Berlin, Germany; E-mail: r.ignatius@labor28.de; Phone: +49-30-82093-262; Fax: +49-30-82093-301. the diagnosis in children in order to avoid the application of invasive tests [9-11]. In addition, SATs are helpful in epidemiological studies $[12,13]$.

The present study aimed at comparing two novel assays (one EIA and one ICA, both using monoclonal antibodies) with a monoclonal chemiluminescence immunoassay (CLIA), which has shown before a sensitivity of $90.1 \%$ and a specificity of 92.4\% in comparison with RUT, histology, and UBT [14].

\section{Materials and Methods}

Patients' Samples. Two hundred sixty-six fresh stool samples with defined CLIA results (42 positives, 219 negatives, and 5 samples with borderline results) and sufficient stool material for further testing were collected between January and May 2018 and stored at $-20{ }^{\circ} \mathrm{C}$. To avoid possible degradation of protein due to multiple freezing and thawing cycles, frozen samples were thawed in proportions and immediately tested in parallel in all 3 assays.

Enzyme Immunoassays. CLIA (Liason ${ }^{\circledR}, H$. pylori SA, DiaSorin, Dietzenbach, Germany), EIA, and ICA (RIDASCREEN $^{\circledR}$ and RIDAQUICK ${ }^{\circledR}$; R-Biopharm AG, Darmstadt, Germany) for the detection of $H$. pylori antigens in stool samples were used according to the manufacturers' instructions.

Statistical Analysis. Since none of the assays could be considered gold standard, and in the absence of data from

This is an open-access article distributed under the terms of the Creative Commons Attribution-NonCommercial 4.0 International License (https://creativecommons.org/licenses/by-nc/4.0/), which permits unrestricted use, distribution, and reproduction in any medium for non-commercial purposes, provided the original author and source are credited, a link to the CC License is provided, and changes - if any - are indicated. 
other (invasive or non-invasive) tests for the detection of H. pylori, we calculated the positive and negative percent agreement (PPA and NPA, respectively) between the assays with $95 \%$ confidence intervals $(95 \% \mathrm{CI})$. In addition, data were statistically analyzed by using McNemar's test and by calculation of Cohen's kappa.

Ethics. Since data were collected for quality enhancement of bacteriological diagnostics, and no analyses were performed other than requested, an approval by a local ethics committee was not applied for. The study is in agreement with the General Data Protection Regulation of the EU.

\section{Results}

In total, 266 stool samples obtained from 260 patients (median age, 45 years; range, 1-96 years) were included in the study. One hundred sixty-four $(61.7 \%)$ of these samples were from female patients, and 102 samples (38.4\%) from male patients.

Thawed stool samples were subjected again to CLIA and simultaneously to EIA and ICA. Initial CLIA results could be replicated by the second CLIA measurements in 249/266 (93.6\%) samples, 207 of these being negative, 41 positive, and 1 sample yielding borderline results twice. One of the 41 CLIA-positive samples was negative by both EIA and ICA, whereas 3 additional samples were negative by the ICA only (Table 1). One and 6 of 207 CLIA-negative samples were EIA-positive or ICA-positive, respectively. The one sample with consistently borderline CLIA results was negative in both EIA and ICA.

Due to sufficient sample material, analysis of 12 samples with questionable ICA results in the first run including CLIAnegative and CLIA-positive samples could be repeated (Table 2). Data for 2 samples each could be either corrected $(116,68)$ or remained false positive or negative, respectively $(194,15)$. For the other 8 samples, the results were slightly

Table 1. Detection of $H$. pylori-specific antigen by ICA, EIA, and CLIA in 248 stool samples with consistent positive or negative CLIA results

\begin{tabular}{|c|c|c|c|}
\hline & & \multicolumn{2}{|c|}{ CLIA } \\
\hline & & Positive & Negative \\
\hline \multirow[t]{2}{*}{ EIA } & Positive & 40 & 1 \\
\hline & Negative & 1 & 206 \\
\hline \multirow[t]{2}{*}{ ICA (initially) } & Positive & 37 & $6^{\mathrm{a}}$ \\
\hline & Negative & 4 & 201 \\
\hline \multirow[t]{2}{*}{ ICA (retested) ${ }^{a}$} & Positive & 38 & 5 \\
\hline & Negative & 3 & 202 \\
\hline
\end{tabular}

${ }^{a}$ Twelve samples with questionable initial ICA results could be retested.

Table 2. Data (for CLIA and EIA given as OD; sorted by OD results of the first CLIA analysis) for 12 samples with replicate ICA data

\begin{tabular}{lccccc}
\hline \multirow{2}{*}{ Sample number } & \multicolumn{2}{c}{ CLIA } & EIA & \multicolumn{2}{c}{ ICA } \\
\cline { 2 - 4 } \cline { 5 - 6 } & Initial & Second & & Initial & Second \\
\hline 116 & 0.01 & 0.17 & 0.002 & + & - \\
194 & 0.01 & 0.32 & 0.004 & + & + \\
20 & 0.39 & $\mathbf{1 . 9 9}$ & $\mathbf{1 . 7 3 9}$ & - & $((+))$ \\
80 & 0.50 & 0.01 & 0.002 & $(+)$ & $(+)$ \\
72 & 0.68 & 0.72 & $\mathbf{0 . 5 8 9}$ & - & - \\
1 & 1.08 & 0.78 & $\mathbf{2 . 0 0 7}$ & - & $((+))$ \\
64 & $\mathbf{2 . 4 8}$ & $\mathbf{3 . 8 3}$ & $\mathbf{1 . 4 1 1}$ & $(+)$ & + \\
69 & $\mathbf{2 . 5 6}$ & $\mathbf{3 . 3 6}$ & $\mathbf{1 . 2 3 2}$ & $(+)$ & + \\
68 & $\mathbf{3 . 2 2}$ & $\mathbf{5 . 4 1}$ & $\mathbf{1 . 5 4 7}$ & - & + \\
26 & $\mathbf{4 . 3 1}$ & $\mathbf{2 . 6 2}$ & $\mathbf{2 . 2 1 2}$ & $((+))$ & $(+)$ \\
15 & $\mathbf{4 . 4 3}$ & $\mathbf{6 . 6 7}$ & $\mathbf{2 . 1 4 2}$ & - & - \\
24 & $\mathbf{1 7 . 1 0}$ & $\mathbf{9 . 7 6}$ & $\mathbf{3 . 4 1 9}$ & $(+)$ & + \\
\multicolumn{2}{c}{${ }^{a}$ Positive results in bold; borderline results highlighted gray. } \\
\hline
\end{tabular}

Table 3. Data (for CLIA and EIA given as OD; sorted by OD results of the first CLIA analysis) for 17 stool samples with discrepant CLIA results

\begin{tabular}{|c|c|c|c|c|}
\hline \multirow[t]{2}{*}{ Sample number } & \multicolumn{2}{|c|}{ CLIA } & \multirow[t]{2}{*}{$\mathrm{EIA}^{a}$} & \multirow[t]{2}{*}{ ICA } \\
\hline & Initial & Second & & \\
\hline 190 & 0.29 & $0.91^{a}$ & 0.001 & - \\
\hline 248 & 0.32 & 1.36 & 0.003 & - \\
\hline 264 & 0.34 & 1.16 & 0.003 & - \\
\hline 20 & 0.39 & 1.99 & 1.739 & $-/((+))^{b}$ \\
\hline 228 & 0.39 & 1.24 & 0.002 & - \\
\hline 186 & 0.41 & 1.01 & 0.003 & - \\
\hline 54 & 0.42 & 0.92 & 0.002 & - \\
\hline 241 & 0.45 & 1.27 & 0.002 & - \\
\hline 145 & 0.58 & 0.91 & 1.271 & - \\
\hline 266 & 0.70 & 1.84 & 0.270 & - \\
\hline 240 & 0.73 & 3.71 & 3.026 & - \\
\hline 252 & 0.80 & 1.68 & 0.001 & - \\
\hline 76 & 0.91 & 0.67 & 0.199 & - \\
\hline 84 & 0.97 & 0.62 & 0.002 & - \\
\hline 143 & 0.99 & 0.27 & 0.001 & - \\
\hline 1 & 1.08 & 0.78 & 2.007 & $-/((+))$ \\
\hline 137 & 1.20 & 0.47 & 0.004 & - \\
\hline
\end{tabular}

modified or repeated as initially seen $(80,72,64,69,26,24)$ or extremely faint bands were visible $(20,1)$, which might have gone undetected without knowing the correct results. Thus, the latter 2 samples were considered ICA-negative. Notably, the EIA-positive sample 72 yielded negative results in both CLIA runs and repeatedly by ICA.

In 17 samples $(6.4 \%)$, discrepant results were obtained between the 2 CLIA measurements (Table 3 ). The vast majority of these were CLIA borderline or low positive (optical density [OD], 0.9-1.99) in either the first or the second CLIA measurement, and only sample 240 was negative in the initial testing but later clearly positive in both the second CLIA testing and the EIA, while the ICA yielded a negative result. Notably, 5 additional samples $(20,145,266,76$, and 1) were also EIApositive, 3 of these with OD $>1$ (Table 2). Initial ICA results were negative for all 17 samples; ICA analysis could be repeated for 2 samples, which then yielded very weakly positive results as mentioned before.

Since no gold standard assay could be included in the study, the overall statistical analysis relied on calculating PPA and NPA and included only the 248 samples with consistent positive or negative CLIA results. Here, we observed high agreement between the CLIA and both EIA and ICA (Table 4). Accordingly, the assays did not differ when compared using the McNemar's test (CLIA vs. EIA, 0.480; CLIA vs. ICA, initial analysis only, 0.752; CLIA vs. ICA including repeat testing, 0.724). Similarly, the kappa values also indicated very good agreement between the assays (CLIA vs. EIA, 0.971; CLIA vs. ICA, initial analysis only, 0.857; CLIA vs. ICA including repeat testing, 0.885 ).

Table 4. Positive and negative percent agreement between CLIA and EIA or ICA, respectively, for 248 samples with consistent positive or negative CLIA results

\begin{tabular}{lcc}
\hline Assay & PPA $^{a}$ & NPA \\
\hline EIA & $97.6 \%$ & $99.5 \%$ \\
& $(86.3-100 \%)$ & $(97.0-100 \%)$ \\
ICA (initial analysis only) & $90.2 \%$ & $97.1 \%$ \\
& $(76.9-96.7 \%)$ & $(93.7-98.8 \%)$ \\
ICA (including repeat testing) & $92.7 \%$ & $97.6 \%$ \\
& $(79.9-98.2 \%)$ & $(94.3-99.1 \%)$
\end{tabular}

${ }^{a} \mathrm{PPA}$, positive percent agreement; NPA, negative percent agreement; data with $95 \% \mathrm{CI}$ in parentheses. 


\section{Discussion}

The present study evaluated for the first time two novel assays for the detection of $H$. pylori antigen in human stool samples. A limitation of the present study already mentioned above is the lack of further data from other invasive or non-invasive tests. In comparison to a previously tested CLIA, however, both ICA and EIA proved to be valuable tools for the laboratory diagnosis of $H$. pylori infection.

Although not statistically significant, the ICA appeared to be slightly less sensitive than the EIA, which, however, is in accordance with previous studies $[3,4]$. We were able to repeat 12 measurements for samples, where the ICA results contradicted the data obtained by the other tests. Clearly, the altered results here observed for 2 samples might be due to pre-analytical issues, e.g., specimens, reagents, and test cassettes must be at room temperature before the analysis, or to an inhomogeneous distribution of antigen within the samples. Notably, very faint bands might be easier to be recognized with some more experience with this assay. As all ICAs, the assay tested is fast and does not require further equipment, such as an ELISA reader.

We observed very high PPA and NPA (kappa value, 0.971) between the EIA and the CLIA, which has a sensitivity of $90.1 \%$ and a specificity of $92.4 \%$ [14]. In comparison to the CLIA, one advantage of the EIA was the very low number of samples yielding OD values close to the cut-off of the assay. In fact, all negative samples yielded OD values at least threefold lower than the cut-off, while the OD values of all but 2 positive samples were at least threefold higher than the cut-off. None of the samples yielded a borderline result. In contrast, one sample yielded a borderline result in both CLIA runs, and we observed 4 samples each with borderline results in one of the 2 runs being negative in the other one. Likewise, a considerable number of samples yielded weakly positive or negative results close to borderline in either CLIA measurement, some of which could not be confirmed in the other CLIA measurement. Thus, the EIA seems to discriminate more precisely between positive and negative samples than the CLIA.

In conclusion, both EIA and ICA can be employed to detect H. pylori antigen in human stool samples. While the ICA is rapid and easy to perform, the EIA demonstrated considerable precision without yielding borderline results.

\section{Funding Sources}

No additional funding was received to perform this study.

\section{Authors' Contributions}

RI, CB, ML, JF, and MM designed the study; CB, CW, $\mathrm{AD}$, and TW performed the experiments; RI and ML analysed the data; RI drafted the manuscript; all authors read and approved the final version of the manuscript.

\section{Conflict of Interest}

ML is employee of R-Biopharm; the other authors declare no conflict of interest.

\section{References}

1. Wang YK, Kuo FC, Liu CJ, Wu MC, Shih HY, Wang SS, et al. Diagnosis of Helicobacter pylori infection: Current options and developments. World J Gastroenterol. 2015;21:11221-35.

2. Bessede E, Arantes V, Megraud F, Coelho LG. Diagnosis of Helicobacter pylori infection. Helicobacter. 2017; Sep 22 Suppl 1.

3. Calvet X, Lario S, Ramirez-Lazaro MJ, Montserrat A, Quesada M, Reeves L, et al. Comparative accuracy of 3 monoclonal stool tests for diagnosis of Helicobacter pylori infection among patients with dyspepsia. Clin Infect Dis. 2010;50:323-8.

4. Korkmaz H, Kesli R, Karabagli P, Terzi Y. Comparison of the diagnostic accuracy of five different stool antigen tests for the diagnosis of Helicobacter pylori infection. Helicobacter. 2013;18:384-91.

5. Gisbert JP, de la Morena F, Abraira V. Accuracy of monoclonal stool antigen test for the diagnosis of $H$. pylori infection: a systematic review and meta-analysis. Am J Gastroenterol. 2006;101:1921-30.

6. Fock KM, Katelaris P, Sugano K, Ang TL, Hunt R, Talley NJ, et al. Second Asia-Pacific Consensus Guidelines for Helicobacter pylori infection. J Gastroenterol Hepatol. 2009;24:1587-600.

7. Malfertheiner P, Megraud F, O'Morain CA, Gisbert JP, Kuipers EJ, Axon AT, et al. Management of Helicobacter pylori infection-the Maastricht V/Florence Consensus Report. Gut. 2017;66:6-30.

8. Fischbach W, Malfertheiner P, Lynen Jansen P, Bolten W, Bornschein J, Buderus S, et al. (S2k-guideline Helicobacter pylori and gastroduodenal ulcer disease]. Z Gastroenterol. 2016;54:327-63.

9. Leal YA, Cedillo-Rivera R, Simon JA, Velazquez JR, Flores LL, Torres J. Utility of stool sample-based tests for the diagnosis of Helicobacter pylori infection in children. J Pediatr Gastroenterol Nutr. 2011;52:718-28.

10. Queiroz DM, Saito M, Rocha GA, Rocha AM, Melo FF, Checkley W, et al. Helicobacter pylori infection in infants and toddlers in South America: concordance between (13C)urea breath test and monoclonal H. pylori stool antigen test. J Clin Microbiol. 2013;51:3735-40.

11. Zhou X, Su J, Xu G, Zhang G. Accuracy of stool antigen test for the diagnosis of Helicobacter pylori infection in children: a meta-analysis. Clin Res Hepatol Gastroenterol. 2014;38:629-38.

12. Shimoyama T, Oyama T, Matsuzaka M, Danjo K, Nakaji S, Fukuda S. Comparison of a stool antigen test and serology for the diagnosis of Helicobacter pylori infection in mass survey. Helicobacter. 2009;14:87-90.

13. Okuda M, Osaki T, Lin Y, Yonezawa H, Maekawa K, Kamiya S, et al. Low prevalence and incidence of Helicobacter pylori infection in children: a population-based study in Japan. Helicobacter. 2015;20:133-8.

14. Ramirez-Lazaro MJ, Lite J, Lario S, Perez-Jove P, Montserrat A, Quilez ME, et al. Good diagnostic accuracy of a chemiluminescent immunoassay in stool samples for diagnosis of Helicobacter pylori infection in patients with dyspepsia. J Investig Med. 2016;64:388-91. 